\title{
The study of the angular and spatial distribution of radio selected AGNs and star-forming galaxies in the ELAIS N1 field
}

\author{
Arnab Chakraborty, ${ }^{1 \star}$ Prasun Dutta, ${ }^{2}$ Abhirup Datta, ${ }^{1}$ Nirupam Roy, ${ }^{3}$ \\ ${ }^{1}$ Discipline of Astronomy, Astrophysics and Space Engineering, Indian Institute of Technology Indore, Indore 453552, India \\ ${ }^{2}$ Department of Physics, IIT (BHU) Varanasi, 221005 India \\ ${ }^{3}$ Department of Physics, Indian Institute of Science,Bangalore 560012,India
}

Last updated 2015 May 22; in original form 2013 September 5

\begin{abstract}
The cosmic evolution of bias of different source populations with underlying dark matter density field in post reionization era can shed light on large scale structures. Studying the angular and spatial distribution of different compact sources using deep radio catalogue at lowfrequency is essential to understand the matter distribution of the present Universe. Here, we investigate the relationship of luminous matter with their host dark matter haloes by measuring the angular and spatial clustering of sources (two-point statistics), using deep radio observation of ELAIS N1 (EN1) field with upgraded Giant Metrewave Radio Telescope (uGMRT) at 300$500 \mathrm{MHz}$. We also analyze the $612 \mathrm{MHz}$ GMRT archival data of the same field to understand the cosmic evolution of clustering of different source populations. We classify the sources as star-forming galaxies (SFGs) and active galactic nuclei (AGN) based on their radio luminosity. We find that the spatial clustering length and bias to the dark matter density field of SFGs are smaller than AGNs at both frequencies. This proves that AGNs are mainly hosted by massive haloes and hence strongly clustered. However, a small decrease in the bias for both kind of sources at higher frequency indicates that we are most likely tracing the faint objects residing in less massive haloes at higher frequencies. Our results are in excellent agreement with previous findings at radio and multi-frequency surveys. However, comparison with SKADS simulation suggests that the halo mass for different populations used in the simulation is systematically lower. This work quantifies the spatial distribution of extragalactic compact objects in EN1 field and bridges the gap between shallow and deep surveys.
\end{abstract}

Key words: galaxies: active âĂŞ galaxies: evolution âĂŞ cosmology: observations âĂŞ large-scale structure of Universe âÂŞ radio continuum: galaxies.

\section{INTRODUCTION}

Observation of the oldest light of the Universe, Cosmic Microwave Background (CMB) radiation, have shown that the early Universe (at $z \sim 1100$ ) was remarkably smooth with tiny anisotropies of the order of $10^{-4}$ (Planck Collaboration et al. 2016). The Universe was almost neutral at that epoch. The linear fluctuations in the matter density grew under gravitational clustering and eventually generated luminous objects. The high energy photons emanating from the first stars and galaxies started heating and ionizing the intergalactic medium (IGM) and forced the Universe to go through a global phase transition. Constraint from Gunn Peterson trough in quasar absorption spectra indicate that the gas in the intergalactic medium (IGM) became completely ionized by redshift $z \sim 6$ (Fan et al. 2002; Fan, Carilli, \& Keating 2006). The transition of the universe from the neutral to ionized phase is called the epoch of

^ phd1601121009@iiti.ac.in reionization (EoR) (Field 1958; Madau 1997; Furlanetto, Oh, \& Briggs 2006; Pritchard \& Loeb 2012; Loeb \& Furlanetto 2013; Mesinger 2016; Barkana 2016; Dayal \& Ferrara 2018)). In the post reionization universe, several astrophysical parameters other than the gravitational clustering influenced the evolution of the baryonic matter density. Estimation of the clustering pattern of different radio sources and comparing it to the dark matter power spectrum holds the key to understand the astrophysical aspects of the post EoR evolution of the matter density distribution.

The extragalactic sky at low-frequency in $\mu \mathrm{Jy}$ to mJy flux limit is mainly dominated by FanaroffâĂŞRiley type I (FR I), radio quiet quasars and star-forming galaxies. One of the possible way to understand these sources along with their host dark matter haloes is through their clustering. The clustering of galaxies can be quantified through counting excess number of galaxies at a certain scale to that of a random distribution of galaxies. This is known as the twopoint correlation function (Lindsay et al. 2014; Hale et al. 2018). Traditionally, the two-point angular correlation function using op- 


\section{Arnab Chakraborty}

tical galaxy surveys are studied and the redshift information is used to estimate the spatial clustering and the bias with the underlying matter power spectrum. However, covering a large fraction of sky is extremely difficult in the optical surveys. Furthermore, dust obscuration limits the detection of high redshifts sources. Emissions in radio wavelengths from these galaxies are not attenuated by dust and hence provide a direct probe of high $z$ galaxies (Jarvis et al. 2016). The relatively larger field of view of the radio telescopes also increase the survey speed. So, deep radio surveys allow to estimate the cross-correlation of the luminous matter distribution with $\mathrm{CMB}$ and shed light on how the baryons traces the underlying dark matter distribution (Planck Collaboration et al. 2014; Allison et al. 2015). Multifrequency observations can be used to understand the relationship between AGNs and star formation processes with their host dark matter haloes (Hale et al. 2018). Previous findings suggest that AGNs are mainly hosted by massive haloes and strongly clustered than SFGs (Gilli et al. 2009; Donoso, et al. 2014; Magliocchetti et al. 2017; Hale et al. 2018). An efficient method to understand the bias of these populations to the underlying dark matter density field is the ratio of spatial correlation lengths of luminous matter and dark matter density field (Peebles 1980). This study of large scale distribution of dark matter through clustering pattern of luminous objects is important to understand the structure formation (Hale et al. 2018). Measurement of bias for different populations of sources in radio surveys allow to probe non-Gaussianity in the initial density fluctuation (Seljak 2009; Raccanelli et al. 2015).

The $21 \mathrm{~cm}$ signal from the EoR and post EoR universe traces the evolution of the matter density over all redshifts. The major hindrance on our quest to detect the redshifted $21 \mathrm{~cm}$ signal are bright dominating foregrounds. Foreground contamination due to diffuse Galactic synchrotron emission from our Galaxy (DGSE) (Shaver et al. 1999), free-free emission from Galactic and extragalactic sources (Cooray \& Furlanetto 2004), faint radio-loud quasars (Di Matteo et al. 2002), synchrotron emission from low-redshift Galaxy clusters (Di Matteo et al. 2004), extragalactic point sources (Di Matteo et al. 2004), etc, are several order of magnitudes higher than the cosmological signal of interest (Zaldarriaga, Furlanetto, \& Hernquist 2004; Bharadwaj \& Ali 2005; Jelić et al. 2008; Bernardi et al. 2009; Jelić et al. 2010; Zaroubi et al. 2012). Di Matteo et al. (2004) have shown that spatial clustering of extragalactic sources with flux densities $\gtrsim 0.1 \mathrm{mJy}$ dominates the angular fluctuation at scales, $\theta \gtrsim 1$ arcmin and modelling and removing these sources will allow us to detect angular fluctuation in $21 \mathrm{~cm}$ emission. However, to understand the systematic effects of the foregrounds on calibration and the effect of bright point sources far away from the field of view of observations, we require an accurate statistical model of the compact source distribution. The extra-galactic source counts are often modelled as single power law or a smooth polynomial (Intema et al. 2017; Franzen et al. 2019) and the spatial distribution of sources is assumed to be Poissonian (Ali, Bharadwaj, \& Chengalur 2008; Trott et al. 2016) or a simple power-law clustering pattern has been implemented. The assumption of simple Poissonian distribution of sources in EoR foreground modelling is too simplistic and will effect the signal extraction gravely (Ali, Bharadwaj, \& Chengalur 2008; Jelić et al. 2010; Ghosh et al. 2012; Trott et al. 2016). This demands a better observational estimate of the clustering pattern of the point sources at the lower radio frequencies.

With this motivation, here we have studied in detail the clustering pattern of different sources (AGNs and SFGs) in EN1 field using deep radio survey with uGMRT, at $300-500 \mathrm{MHz}$. Along with that, to understand the redshift evolution of bias of different sources we also study the clustering of different populations using $612 \mathrm{MHz}$
Table 1. Details of different parameters of radio data used in this work

\begin{tabular}{ccccc}
\hline \hline Target & $\begin{array}{c}v \\
(\mathrm{MHz})\end{array}$ & $\begin{array}{c}S_{\text {limit, } v} \\
\left(\mu \mathrm{Jy} \mathrm{beam}^{-1}\right)\end{array}$ & $\begin{array}{c}\text { Survey area } \\
\left(\mathrm{deg}^{2}\right)\end{array}$ & $\begin{array}{c}\text { Total sources } \\
(\mathrm{N})\end{array}$ \\
\hline EN1 & 400 & 100.0 & 1.8 & 2528 \\
\hline EN1 & 612 & 50.0 & 1.13 & 2342 \\
\hline
\end{tabular}

$\dagger S_{\text {limit }, v}$ is the flux density limit of the corresponding catalogue at observed frequency $(v)$.

GMRT archival data of the same field. In our previous works, we have studied the spatial and spectral properties of diffuse galactic synchrotron emission, the angular power spectrum of Galactic and extragalactic foregrounds, the differential source counts as a function of flux densities, etc in the same field (Chakraborty et al. 2019a,b).

The paper is structured as follows. The details of radio and optical data used in this work is presented in Sec. 2. The preprocessing of the source catalogues to estimate the angular and spatial clustering of sources and the classification of sources based on radio luminoisty is discussed in Sec. 3. The estimated angular correlation of all sources and a detailed comparison with previous findings are presented in Sec. 4.1. The results of spatial clustering and bias of the sources to the dark matter density field is discussed in Sec. 5. The clustering pattern of AGNs and SFGs along with the detail comparison with previous observations and simulations are discussed in Sec. 6. Finally, we draw conclusion in Sec. 7.

Throughout this work, we use the best fitted cosmological parameters of the Planck 2018 analysis (Planck Collaboration et al. 2018), which are: $\Omega_{\mathrm{M}}=0.31, \Omega_{\Lambda}=0.68, \sigma_{8}=0.811, H_{0}=67.36$ $\mathrm{Km} \mathrm{s}^{-1} \mathrm{MPc}^{-1}$

\section{SOURCES OF DATA AT DIFFERENT FREQUENCY BANDS}

We use $400 \mathrm{MHz}$ uGMRT observation of EN1 field along with archival GMRT $612 \mathrm{MHz}$ data for cataloguing radio sources at these frequency bands. In this section, we briefly discuss the radio and optical data used in this work to estimate the angular and spatial clustering properties of sources in the EN1 field. The details of radio surveys are mentioned in Table 1.

\section{1 uGMRT observation at $400 \mathrm{MHz}$}

The EN1 field $\left(\alpha_{2000}=16^{h} 10^{m} 1^{s}, \delta_{2000}=54^{\circ} 30^{\prime} 36^{\prime \prime}\right)$ has been observed with uGMRT in GTAC cycle 32 during May 2017 at 300 - $500 \mathrm{MHz}$ for a total on source time of 25 hours. The raw data has a time resolution of $2 \mathrm{sec}$ and frequency resolution of $24 \mathrm{KHz}$. This high resolution data was flagged for RFI with AOFLAGGER (Offringa, van de Gronde, \& Roerdink 2012) and then averaged to $16 \mathrm{sec}$ time and $97 \mathrm{kHz}$ frequency resolution for further processing. The calibration, imaging and self-calibration was done in CASA 1 (Chakraborty et al. 2019b). The final image, covering $\sim 1.8 \mathrm{deg}^{2}$ area, has a off-source noise of $\sim 15 \mu \mathrm{Jy}_{\text {beam }}{ }^{-1}$ in the central part ( Fig. 1: left panel). A source catalogue of 2528 sources with flux densities above $100 \mu \mathrm{Jy}$ ( $>6 \sigma$ ) was compiled using PyBDSF (Mohan \& Rafferty 2015). In this work we use these catalogued

\footnotetext{
1 See: https://casa.nrao.edu; (McMullin et al. 2007)
} 
compact radio sources in the EN1 field to estimate the angular and spatial clustering (the catalogue is publicly available ${ }^{2}$ ). The detail analysis of the uGMRT $400 \mathrm{MHz}$ observation, calibration, source extraction and catalogue formation is presented in Chakraborty et al. (2019b).

\subsection{GMRT archival data at $612 \mathrm{MHz}$}

We additionally use the GMRT archival data of EN1 field, observed between 2011 to 2013 at $612 \mathrm{MHz}$ (project codes: 20_044, 21_083, 22_056) in this study. We re-analyze this GMRT archival data and briefly mention the procedure here. Observations were carried out using seven different pointings arranged in a hexagonal pattern centered at $\alpha_{2000}=16^{h} 10^{m} 30^{s}$., $\delta_{2000}=54^{\circ} 35^{\prime} 00^{\prime \prime}$ (Taylor \& Jagannathan 2016). We perform necessary flagging and calibration of each pointing using the AIPS based automated pipeline Source peeling and Atmospheric Modeling (SPAM) (Intema et al. 2009, 2017). We create a mosaic of the seven pointings to create a combined image of the field which covers $\sim 1.13 \mathrm{deg}^{2}$ area. We achieve a off-source rms of $\sim 8 \mu \mathrm{Jy}_{\text {beam }}{ }^{-1}$ at the central part of the image (Fig. 1: right panel). We use PyBDSF on the final image to compile a $612 \mathrm{MHz}$ source catalogue of the EN1 field having 2342 sources with flux densities above $50 \mu \mathrm{Jy}(>6 \sigma)$.

\subsection{BOSS spectroscopic survey}

The Baryon Oscillation Spectroscopic Survey (BOSS) is the dark time survey of the third phase of the Sloan Digital Sky Survey or SDSS-III (SDSS; York et al. 2000; Dawson et al. 2013; Alam et al. 2015). This survey primarily aims to determine Baryon Acoustic Oscillation feature (BAO) in large scale structure (Cole et al. 2005; Eisenstein et al. 2005). It has measured redshift of 1.5 million galaxies and have obtained distance-redshift relation $d_{A}(z)$ and the Hubble parameter $H(z)$ with highest precision till date. Four plates were granted to observe spectra of the radio sources identified in the EN1 field by LOFAR, GMRT, JVLA and FIRST. After data reduction, spectra of sources observed in each plate along with their position and redshift is made available as a part of SDSS DR12. We use this spectroscopic redshift catalogue released by SDSS team to identify the redshift of the radio sources in our catalogues discussed above $^{3}$ (Alam et al. 2015).

\subsection{SWIRE photometric survey}

The revised Spitzer Wide-Area Infrared Extragalactic survey (SWIRE) catalogue incorporates Two Micron All Sky Survey (2MASS) and UKIRT Infrared Deep SkySurvey (UKIDSS) nearinfrared data, which essentially reduces the fraction of catastrophic outliers and gives a more reliable photometric catalogue (RowanRobinson et al. 2008; Rowan-Robinson, Gonzalez-Solares, Vaccari $\&$ Marchetti 2013). The SWIRE survey covers a sky of $49 \mathrm{deg}^{2}$ at 3.6, 4.5, 5.8, 8.0, 24.0, 70.0 and $160.0 \mu m$ using Spitzer. This survey covers $\sim 8.72 \mathrm{deg}^{2}$ of EN1 field in five bands $\left(U^{\prime}, g^{\prime}, r^{\prime}, i^{\prime}, Z^{\prime}\right)$. Together with this, the additional information of JHK magnitude from 2MASS and UKIDSS results in the most reliable photometric redshift catalogue publicly available for this field.

\footnotetext{
2 http://vizier.u-strasbg.fr/viz-bin/VizieR?-source=J/ MNRAS $/ 490 / 24$

${ }^{3}$ https://www.sdss.org/dr12/algorithms/ancillary/boss/ sdsslofar/
}

We use the spectroscopic redshifts in the SDSS catalogue and the photometric redshifts in the SWIRE catalogue complimentarily to get redshifts of the sources identified in the radio frequencies.

\subsection{Semi-empirical extra galactic radio source catalogue}

Wilman et al. (2008) performed semi-empirical simulation to generate extragalactic radio continuum sky where they model the statistical distribution of different sources to be seen in radio continuum with the next generation radio telescope. Their conical simulation volume covers a sky area of $20 \times 20 \mathrm{deg}^{2}$ to a redshift of $z=20$ with sources to a flux density limit of $10 \mathrm{nJy}$. They use five distinct source types, the radio quiet quasars (RQQ), the radio loud AGNs with FRI and FRII morphology, the star forming galaxies with quiescent and starbursts (SB). They use a numerical Press-Schecter (Press \& Schechter 1974) formalism to identify high density halos in the dark matter distribution. A source type is then identified with the given halo mass and the luminosity of the source drawn from an observed luminosity function of the given source type. In this work we use the corresponding source catalogue, statistical inference of the variation of angular and spatial clustering of different source types over the redshift. We shall use $S^{3}$ simulation or $S^{3}$ (SKA Simulated Skies) to denote results of this simulation henceforth.

\section{PREPROCESSING OF THE SOURCE CATALOGUE}

To generate radio catalogue at $400 \mathrm{MHz}$ and $612 \mathrm{MHz}$ with redshift information and source characteristics we proceed with the steps given in this section. These catalogues are used to estimate the angular and spatial clustering of the radio sources.

\subsection{Merging multi-component sources}

In a high resolution radio map, such as here $\left(\sim 5^{\prime \prime}\right)$, many sources resolve into multiple components. These are mostly radio galaxies with a central nucleus; accompanied by hotspots along or at the end of, one or two jets (Prandoni et al. 2018). As a result, a single radio source may split into multiple sources in the catalogue (Magliocchetti, Maddox, Lahav \& Wall 1998). It is important to identify these separate components as a single source to unbiasedly quantify the angular clustering. Oort (1987) finds a strong correlation between the angular size $\theta$ of the radio sources with their flux densities $S$. This relation, $\theta \propto \sqrt{S}$, usually known as the $\theta-S$ relation, is used to identify multiple resolved components of radio sources in various earlier surveys (Magliocchetti, Maddox, Lahav \& Wall 1998).

We use the following two criteria to associate multiple nearby objects in our initial catalogue as a single source in the final catalogue. We first estimate the total flux $S_{\text {total }}$ and angular separations $\theta$ of each pairs of sources and identify the pairs to a maximum angular separation $\theta_{\max }$. The choice of maximum separation is bard on the $\theta-S$ relation discussed above and is given as $\theta_{\max }=20\left(S_{\text {total }}\right)^{0.5}$, where $\theta_{\max }$ is given in arcsec and $S_{\text {total }}$ in mJy (Huynh, Jackson, Norris \& Prandoni 2005; Prandoni et al. 2018). We consider the above identified pairs to come from a single source if their flux densities differ by a factor of less than 4 (Huynh, Jackson, Norris \& Prandoni 2005). Fig. 2 shows the summed flux of each nearest pair as a function of distance between them in black filled circles. The pairs above the blue dashed line have angular separation lesser than $\theta_{\max }$. Sources satisfying both the criteria discussed above are shown as red triangles and are considered as single sources in the 

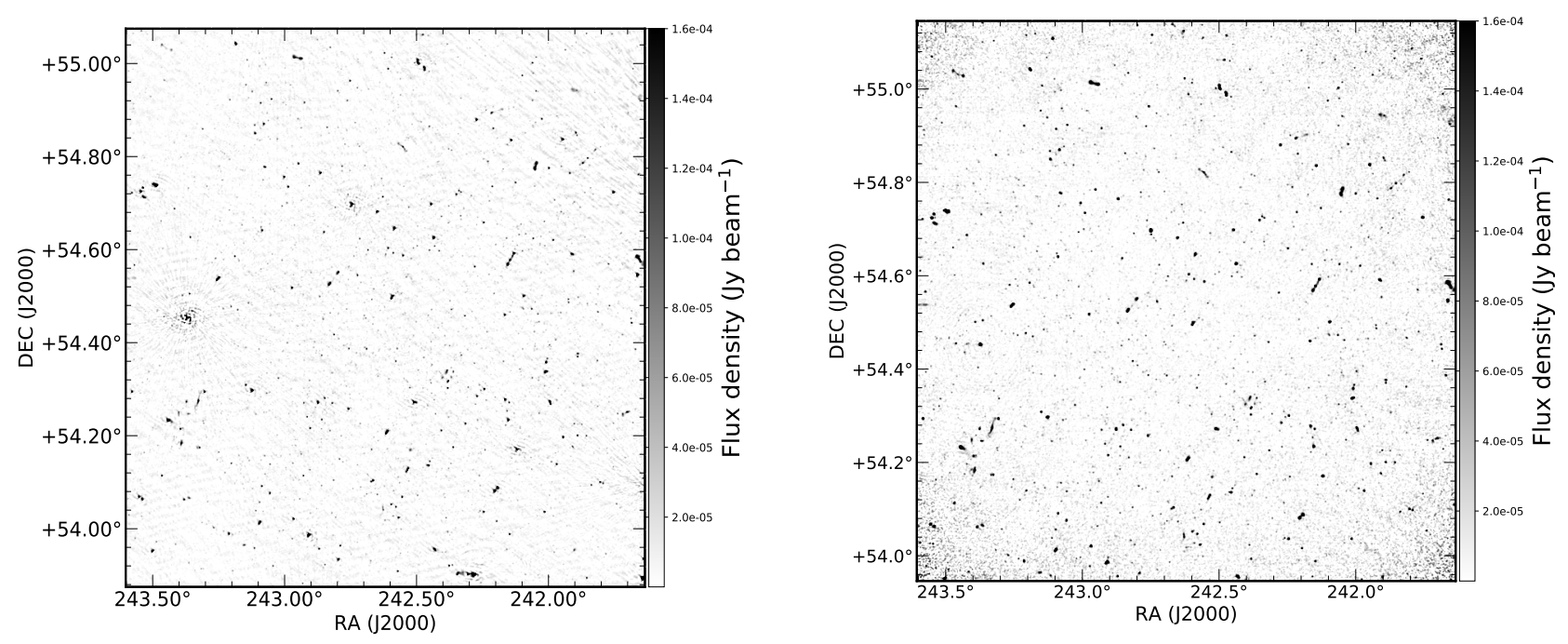

Figure 1. The central $1.2 \mathrm{deg}^{2}$ of total intensity map of the ELIAS N1 at $400 \mathrm{MHz}$ (left) and $612 \mathrm{MHz}$ (right) are shown. The off-source rms at the center is $15 \mu \mathrm{Jy}$ beam $^{-1}$ for $400 \mathrm{MHz}$ map and $\sim 8 \mu \mathrm{Jy}_{\text {beam }}{ }^{-1}$ for $612 \mathrm{MHz}$ map.
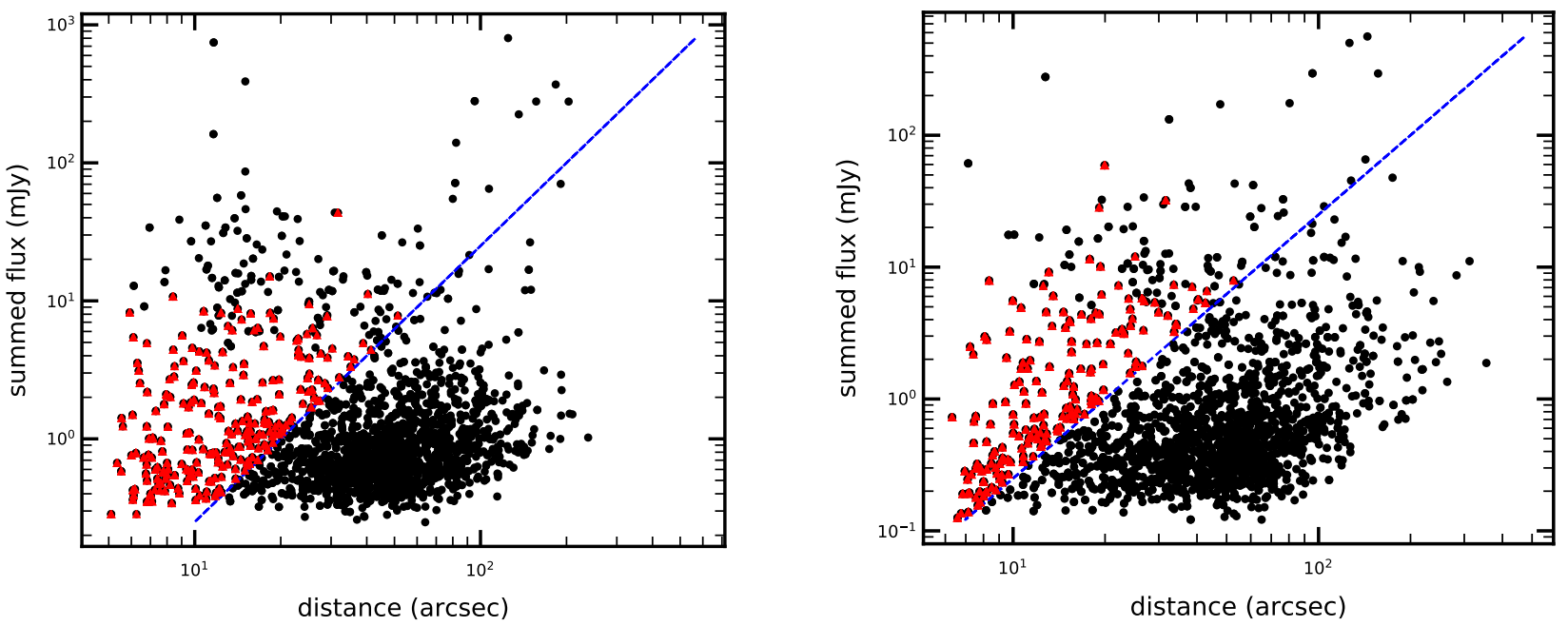

Figure 2. Sum of flux densities of nearest neighbours pairs as a function of their angular separation is plotted in black circles. The pair of sources lying above the blue dashed line and with flux density difference less than by a factor of 4 are shown in red. Left panel and right panel are for $400 \mathrm{MHz}$ and $612 \mathrm{MHz}$ catalogue, respectively.

final catalogue. Using this method we have 2253 and 2153 sources in our final radio catalogue at $400 \mathrm{MHz}$ and $612 \mathrm{MHz}$ respectively.

\subsection{Adding Redshift information of sources}

We use the BOSS spectroscopic survey and the SWIRE photometric survey for optical identification of sources in our radio catalogue. The positional accuracy of the uGMRT observation is less than $1^{\prime \prime}$ (Chakraborty et al. 2019b). Hence, we use a simple nearestneighbour match between the sources in optical and radio catalogue. The search is performed within a radius of $r_{\mathrm{s}}$ from the sources. Lindsay et al. (2014) show that the rate of contamination $P_{\mathrm{c}}$ of the remaining sources due to closeness to optical sources can be expressed as:

$P_{\mathrm{c}}=\pi r_{\mathrm{s}}^{2} \sigma_{\mathrm{opt}}$,

where $r_{\mathrm{s}}$ is the search radius cut-off and $\sigma_{\mathrm{opt}}$ is the surface density of optical catalogue. We have chosen $r_{\mathrm{s}}$ as $3^{\prime \prime}$. For $\sigma_{\mathrm{opt}}=1.4 \times$ $10^{4} \mathrm{deg}^{-2}$, a $3^{\prime \prime}$ search radius results in a contamination rate of $3.1 \%$. This search radius allows us to include a large number of radio samples as well as ensures valid optical identification. We use this search radius for cross-matching.

We find $761(30 \%)$ and $822(35 \%)$ sources with spectroscopic redshifts and $1470(58 \%)$ and $1391(59 \%)$ sources with photometric redshifts for the $400 \mathrm{MHz}$ and $612 \mathrm{MHz}$ catalogues respectively. Fig. 3 shows the correlation plots to compare the photometric and 

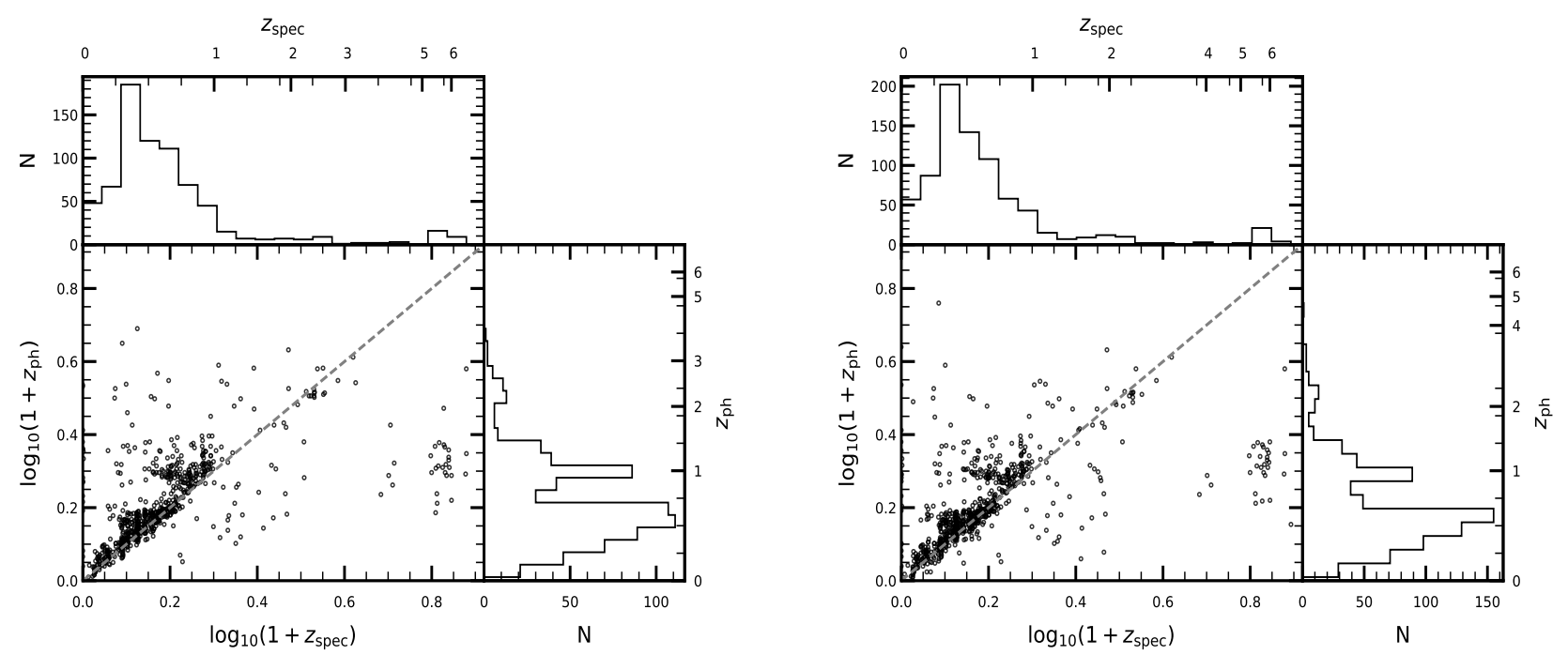

Figure 3. The comparison between photometric and spectroscopic redshift of sources with both for $400 \mathrm{MHz}$ (left panel) and $612 \mathrm{MHz}$ (right panel) catalogue. The histogram of redshifts are also plotted here. The redshift distribution of sources for both the catalogues are nearly identical.

spectroscopic redshifs for both the catalogues respectively. Note that, spectroscopic and photometric redshifts are consistent for low redshift sources for both the catalogues. We also show the histogram of spectroscopic and photometric redshifts for both the catalogues in Fig 3. We associate redshift information to a total of 1503 (59\%) sources for $400 \mathrm{MHz}$ catalogue and 1429 (61\%) sources for 612 $\mathrm{MHz}$ catalogue. We use photometric redshift only for those sources, which do not have spectroscopic redshift information available.

Also, we estimate the outlier fraction defined as, $\frac{\left|z_{\mathrm{ph}}-z_{\mathrm{spec}}\right|}{\left(1+z_{\mathrm{spec}}\right)}>$ 0.2 (Duncan et al. 2018), to asses the precision of photometric redshifts. The outlier fraction makes up $\sim 13 \%$ of the total samples in both cases. In Fig. 4, we show the variation of $\frac{\left|z_{\mathrm{ph}}-z_{\text {spec }}\right|}{\left(1+z_{\text {spec }}\right)}$ as a function of spectroscopic redshifts. We find that for both cases, $400 \mathrm{MHz}$ and $612 \mathrm{MHz}$ catalogue, the estimate of photometric redshifts have some outliers with respect to spectroscopic redshifts. We find that the standard deviation of outlier fraction is $\sim 0.6$ for both catalogues. However, neglecting those outliers from the analysis does not change our statistical estimation of angular and spatial correlation functions. The reason behind large deviation of photometric redshift with respect to spectroscopic redshift for some sources is unknown. In future work we will use more updated redshift catalogue for this particular field.

\subsection{Classification of sources}

In this work we classify the catalogued sources at $400 \mathrm{MHz}$ and 612 $\mathrm{MHz}$ with redshift information as AGNs or SFGs using their radio luminosity as a sole indicator. McAlpine et al. (2013) show that the luminosity function evolves as $(1+z)^{2.5}$ for SFGs but for AGN powered galaxies as $(1+z)^{1.2}$. Further, Magliocchetti et al. (2014, 2016, 2017) showed that AGN powered galaxies become dominant beyond the radio power $P_{\text {cross }}(z)$, which scales with redshift $z$ as :

$\log _{10} P_{\text {cross }}=\log _{10} P_{0, \text { cross }}+z$

to $z \sim 1.8$, where radio power $P$ is in $\mathrm{WHz}^{-1} \mathrm{sr}^{-1}$. In the local Universe $P_{0, \text { cross }}=10^{21.7}\left[\mathrm{WHz}^{-1} \mathrm{sr}^{-1}\right]$ and nearly coincides with the break in the radio luminosity function of star-forming galaxies (Magliocchetti et al. 2002). Beyond that the luminosity function of SFGs decreases rapidly and their population is drastically reduced. The possibility of contamination between the AGNs and SFGs using a luminosity based selection is quite low (Magliocchetti et al. 2017).

The radio luminosities of sources in EN1 field with redshift information can be calculated from their flux using (Magliocchetti et al. 2014):

$P_{1.4 \mathrm{GHz}}=S_{1.4 \mathrm{GHz}} D^{2}(1+z)^{3+\alpha}$,

where $P_{1.4 \mathrm{GHz}}$ is in $\left[\mathrm{WHz}^{-1} \mathrm{sr}^{-1}\right]$ units, $D$ is the angular diameter distance and $\alpha$ is the spectral index of radio emission $\left(S_{v} \propto v^{-\alpha}\right)$. Note that, we do not have pre-measured spectral index values for all the sources in our catalogue. Chakraborty et al. (2019b) compared the flux densities of matched sources in EN1 field with high frequency catalogues and found a median spectral index of 0.8 . Since we are probing the faint sources here, the probability of finding a large number of bright, flat spectrum AGNs is low (Magliocchetti et al. 2017). We consider the spectral index for all the sources as 0.8 to estimate their radio luminosities.

We classify the catalogued sources with redshifts $z \leqslant 1.8$ as AGNs if their luminosity is greater than the threshold given in Eqn. 2 and SFGs otherwise. For higher redshifts, we fix $P_{0 \text {,cross }}=10^{23.5}$ $\left[\mathrm{WHz}^{-1} \mathrm{sr}^{-1}\right.$ ] (McAlpine et al. 2013). Following this procedure, out of 1503 sources at $400 \mathrm{MHz}$ (with redshift information), 314 (20.9\%) and 1189 (79.1\%) sources has been identified as AGNs and SFGs respectively. Whereas, at $612 \mathrm{MHz}$, out of 1429 sources (with redshifts) $290(20 \%)$ and $1139(79 \%)$ sources has been identified as AGNs and SFGs respectively. The redshift distributions of AGNs and SFGs are shown in Fig. 5. The median value of redshift for SFGs is 0.64 and that for the AGNs is 0.91 at $400 \mathrm{MHz}$. For 612 $\mathrm{MHz}$ catalogue the median redshift for SFGs is 0.57 and for AGNs it is 0.85 . 

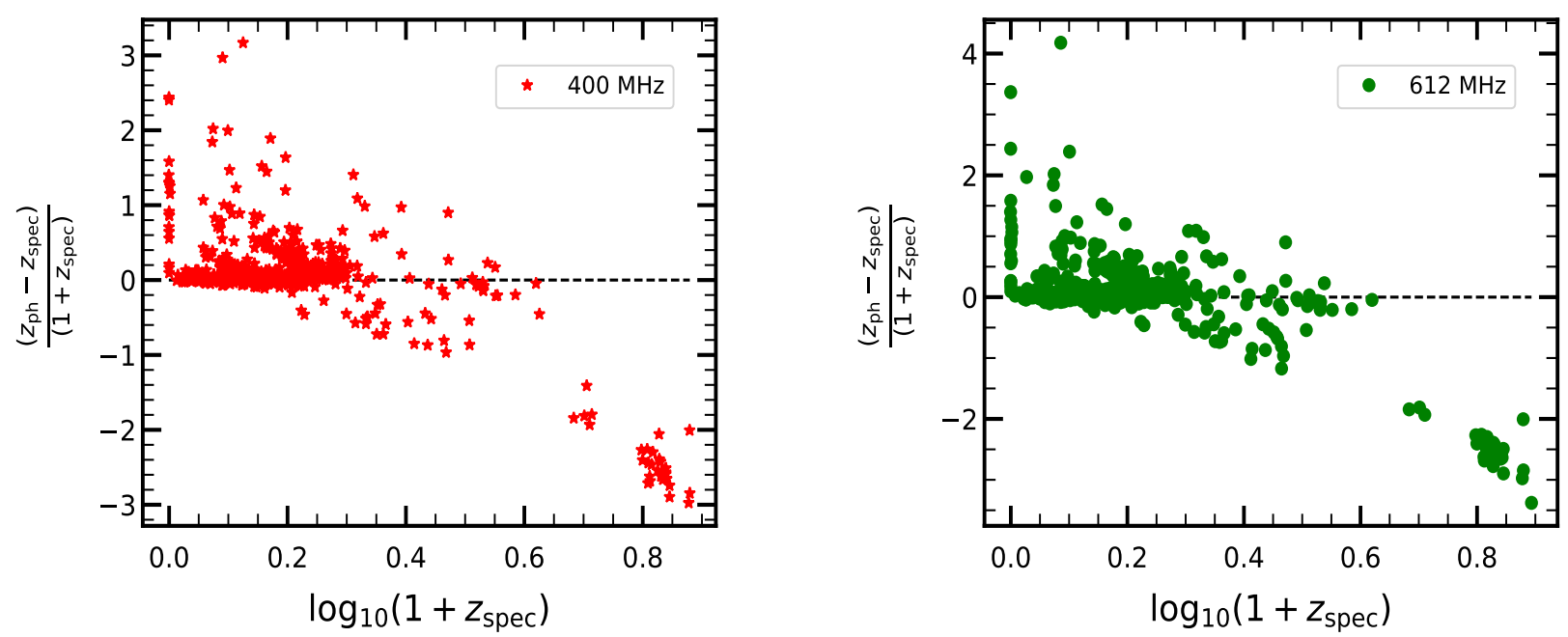

Figure 4. The figure shows the distribution $\frac{\left(z_{\mathrm{ph}}-z_{\mathrm{spec}}\right)}{\left(1+z_{\mathrm{spec}}\right)}$ as a function of $z_{\text {spec }}$. The black dashed line represent zero deviation of photometric redshift with respect to spectroscopic redshift.

\section{ESTIMATION OF THE CORRELATION FUNCTIONS}

\subsection{The angular correlation function}

The angular two-point correlation function, $w(\theta)$, quantifies clustering of sources on angular scale in sky-plane. A detailed discussion on different estimators of $w(\theta)$ can be found in literature (see: Kerscher, Szapudi \& Szalay 2000). In this work, we use the LS estimator proposed by Landy \& Szalay (1993):

$w(\theta)=\frac{D D(\theta)-2 D R(\theta)+R R(\theta)}{R R(\theta)}$.

Here, $D D(\theta)$ and $R R(\theta)$ are the average count of pair of objects separated by an angle $\theta$ in original catalogue and in a random catalogue, respectively. $R R(\theta)$ is calculated from a large simulated catalogue constructed by distributing sources randomly across the same survey area as the real data. This estimator also includes the count of cross-pair separation, $D R(\theta)$, between real and random catalogue, which effectively reduces the large scale uncertainty in source density (Cress et al. 1996; Overzier et al. 2003).

The error in $w(\theta)$ is being calculated using 'bootstrap' resampling method (Ling, Frenk \& Barrow 1986). Here, we have generated 100 'bootstrap' to quote uncertainty in $w(\theta)$ measurement.

\subsection{Random Catalogue}

The noise is not uniform across the map of the EN1 field. This results in systematic non detection of fainter sources at the regions of the field of view with higher noise and hence introduces a bias in the estimate of the angular two point correlation function. The effect of inhomogeneous noise in the image needs to be incorporated in the generation of random catalogue to avoid this bias. We use the following procedure to generate random catalogue in order to estimate the angular correlation function unbiasedly.

We use PyBDSF to estimate the noise map for the EN1 field. Assuming that the radio sources follow the flux distribution of $S^{3}$ catalogue (Wilman et al. 2008), we randomly choose 1000 sources from the $S^{3}$ catalogue with flux densities in a given range. We simulate a radio map with compact sources with the above scaled flux densities distributed randomly in the EN1 field. We generate a mock sky map with these sources and add the above noise map. We use PyBDSF with same parameters as that used to estimate the EN1 catalogue (see Sec.4 in Chakraborty et al. 2019b) to construct the random catalogue from this map. This gives us one realization of the random catalogue that can be used to estimate the angular correlation function unbiasedly. We use 100 statistically independent realizations of such random catalogues to reduce the statistical uncertainty associated with it.

Since the noise map of the $400 \mathrm{MHz}$ and $612 \mathrm{MHz}$ data are different we generated separate random catalogues for them following the procedure described above. For the $400 \mathrm{MHz}$ catalogue we draw sources with flux densities in the range between $75 \mu \mathrm{Jy}$ to 1 Jy from the $S^{3}$ catalogue, whereas for the $612 \mathrm{MHz}$ catalogue the sources were selected within flux density range of $40 \mu \mathrm{Jy}$ to $1 \mathrm{Jy}$, where lower flux density threshold corresponds to the $5 \sigma$ limits of the corresponding maps.

\subsection{Results of clustering pattern of compact sources at 400 MHz and $612 \mathrm{MHz}$}

We use the publicly available code TreeCorr ${ }^{4}$ (Jarvis et al. 2004) to find the angular correlation function of the sources in the 400 $\mathrm{MHz}$ catalogue in 15 equal logarithmically spaced bins between $\theta \sim 18^{\prime \prime}-1.5^{\circ}$. The lower limit for the correlation is chosen to be 4 times the synthesized beam (PSF). Note that the synthesized beam at $612 \mathrm{MHz}$ is higher than $400 \mathrm{MHz}$. This is mainly because of unavailability of large baselines at $612 \mathrm{MHz}$ due to calibration and editing. The upper limit is restricted to the $\sim 1.5$ times the HPBW (primary beam) at respective frequencies. The left panel of Fig. 6, shows the estimated value of $w(\theta)$ at $400 \mathrm{MHz}$ as a function of $\theta$ with (orange) filled circles. The error bars are estimated by 'bootstrap'

\footnotetext{
${ }^{4}$ https://github.com/rmjarvis/TreeCorr
} 

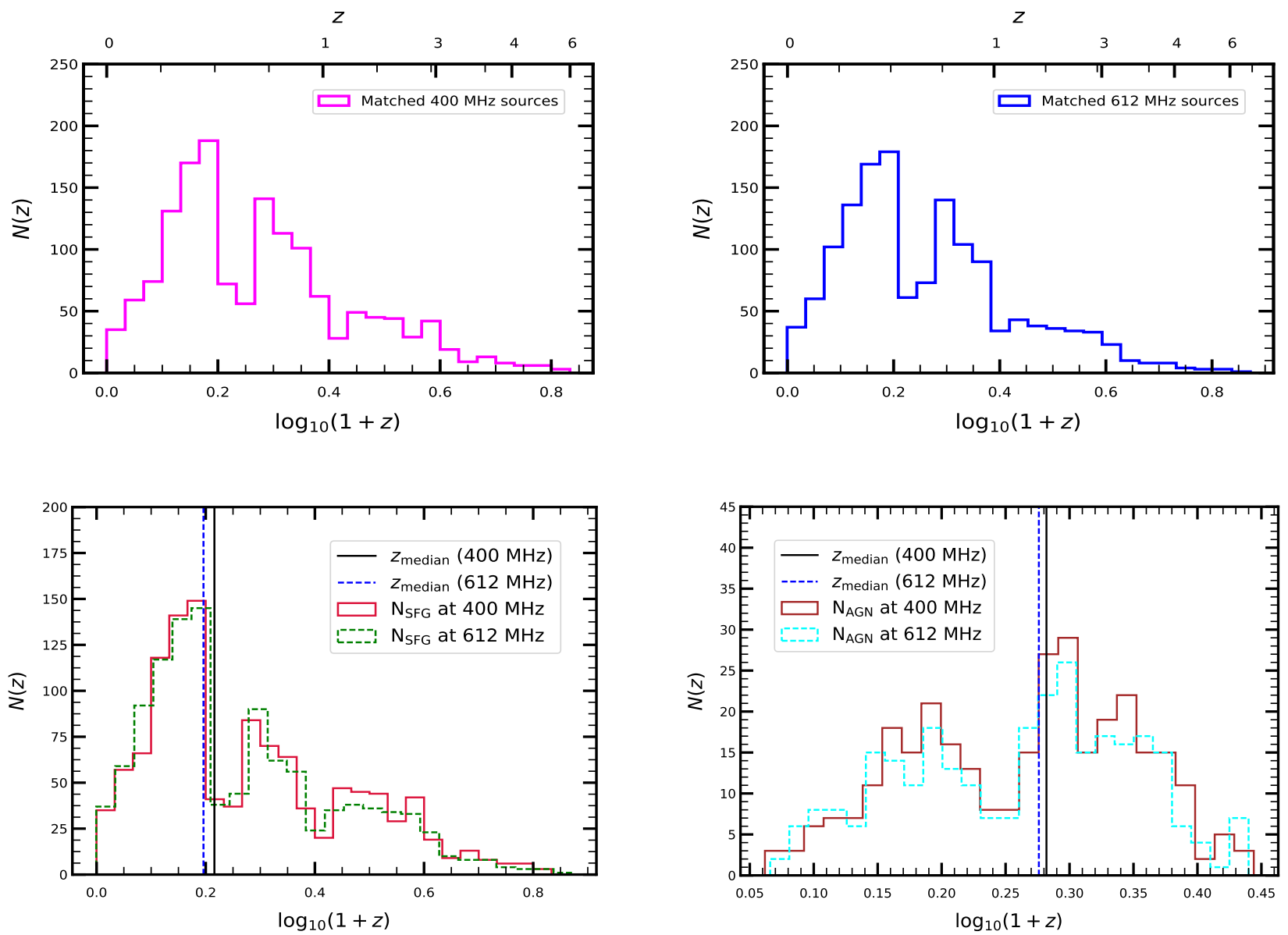

Figure 5. The redshift distribution ( $\mathrm{N}(z)$ ) of matched sources with the SWIRE and SDSS catalogues for $400 \mathrm{MHz}$ (top left) and $612 \mathrm{MHz}$ (top right) catalogue respectively. The redshift distribution of SFGs (bottom left) and AGNs (bottom right) at $400 \mathrm{MHz}$ and $612 \mathrm{MHz}$ are also shown. Here the redshift of sources includes both photometric and spectroscopic redshifts. The black and blue dashed lines in the bottom panel are the median values of the redshift distribution at the corresponding frequency.

Table 2. Clustering parameters from this study and previous obsevations

\begin{tabular}{cccccccc}
\hline \hline Observation & $v(\mathrm{MHz})$ & $S_{\text {limit }, v}^{\dagger}(\mathrm{mJy})$ & $\log _{10}(\mathrm{~A})$ & $\gamma$ & $S_{\text {cut }, 400 \mathrm{MHz}}(\mathrm{mJy})$ & $S_{\mathrm{cut}, 612 \mathrm{MHz}}(\mathrm{mJy})$ & Ref \\
\hline FIRST & 1400 & 1 & $-2.30_{-0.9}^{+0.7}$ & $1.82 \pm 0.02$ & 2.72 & 1.93 & Lindsay et al. (2014) \\
\hline COSMOS & 3000 & 0.013 & $-2.83_{-0.1}^{+0.1}$ & 1.80 & 0.065 & 0.046 & Hale et al. (2018) \\
\hline TGSS & 150 & 50 & $-2.11_{-0.3}^{+0.3}$ & 1.82 & 22 & 16.0 & Rana \& Bagla (2019) \\
\hline XMM-LSS & 144 & 1.4 & $-2.08_{-0.04}^{+0.05}$ & 1.80 & 0.61 & 0.44 & Hale et al. (2019) \\
\hline EN1 & 400 & 0.100 & $-2.03_{-0.08}^{+0.10}$ & $1.75 \pm 0.06$ & 0.100 & 0.071 & This work \\
\hline EN1 & 612 & 0.050 & $-2.22_{-0.15}^{+0.12}$ & $1.81 \pm 0.06$ & 0.070 & 0.050 & This work \\
\hline
\end{tabular}

$\dagger S_{\text {limit }, v}$ is the flux density limit of the corresponding catalogue at observed frequency $(v)$.

re-sampling method as described earlier. We fit $w(\theta)$ with a power law of the form $w(\theta)=A \theta^{1-\gamma}$. We use Markov Chain Monte Carlo (MCMC) simulation for this fitting and parameter estimation. We use Metropolis-Hastings algorithm to simulate $10^{6}$ data points in the $A-\gamma$ parameter space and use $\chi^{2}$ to estimate the most likely values of the parameters. The best fitted values are $\log (\mathrm{A})=-2.03_{-0.10}^{+0.08}$ and $\gamma=1.75 \pm 0.06$, where the error bars are $1-\sigma$ error bars corresponding to $68.3 \% \mathrm{MCMC}$ points.

The right panel of Fig. 6 shows the angular correlation function at $612 \mathrm{MHz}$ as estimated in 15 equally spaced logarithmic bins between $\theta \sim 25^{\prime \prime}-1.2^{\circ}$. The best fit values of the parameters are $\log (\mathrm{A})=-2.22_{-0.15}^{+0.12}$ and $\gamma=1.81 \pm 0.06$. 

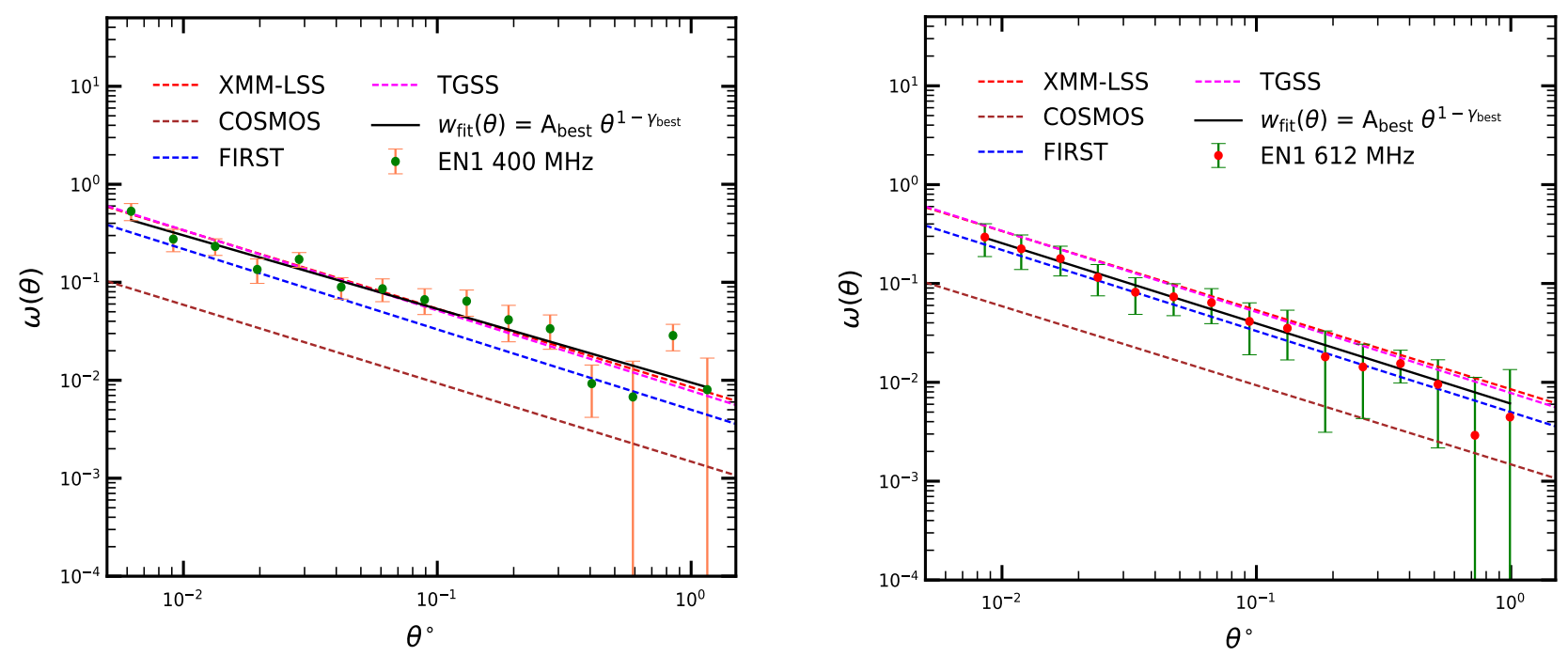

Figure 6. Angular correlation function of sources in EN1 using $400 \mathrm{MHz}$ (left) and $612 \mathrm{MHz}$ (right) catalogue. The black line is the best fitted power law to $w(\theta)$. We also compare our findings with previous observations of XMM-LSS (Hale et al. 2019), FIRST (Lindsay et al. 2014), COSMOS Hale et al. (2018) and TGSS (Rana \& Bagla 2019).

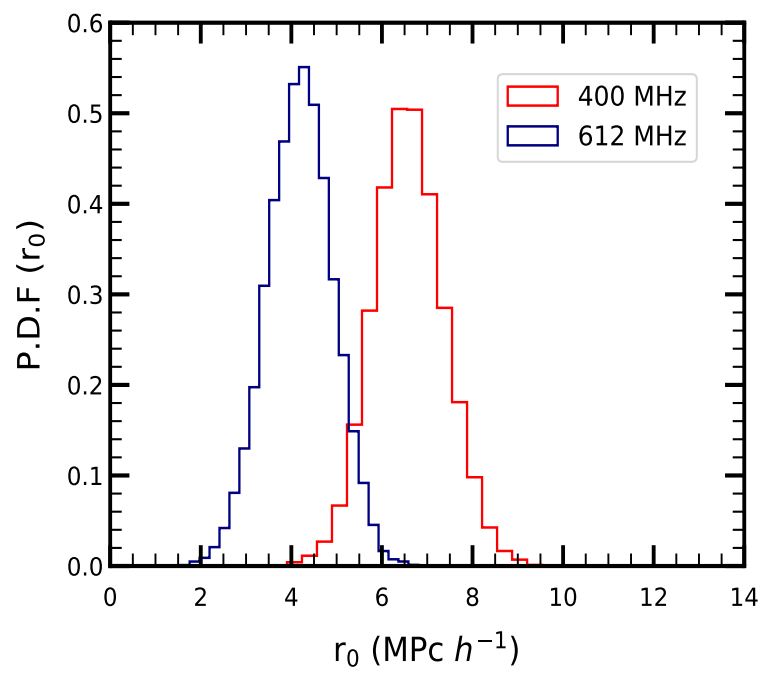

Figure 7. The probability distribution function (PDF) of the spatial clustering length $\left(r_{0}\right)$ for $400 \mathrm{MHz}($ red) and $612 \mathrm{MHz}$ (blue) catalogues.

\subsection{Comparison to previous observations}

The results of angular clustering from various studies along with the findings in this paper is summarized in Table 2. Column (7) and (8) in the Table 2 gives the projected flux density cut offs at 400 and $612 \mathrm{MHz}$ (assuming a spectral index of $0.8 ; S_{v} \propto v^{-\alpha}$ ) for different catalogues used to calculate the angular correlation function. The best fit estimate of the parameter $\gamma$ is found to be consistent with all the previous observations at different frequency bands as well as with its theoretical prediction (Peebles 1980).

Given similar types of sources in a population, the less luminous sources are expected to be found in weakly clustered less massive haloes. This results in measurement of lower value of the amplitude of the correlation function for deeper catalogues. We believe this the reason of higher clustering amplitude in the $400 \mathrm{MHz}$ catalogue as compared to the $612 \mathrm{MHz}$ catalogue.

Hale et al. (2018) measured angular two-point correlation function of radio sources in Cosmological Evolution Survey (COSMOS) field at $3 \mathrm{GHz}$ (Smolčić et al. 2017A,B). Hale et al. (2018) reports estimation of clustering amplitude of all sources as $\log (\mathrm{A})=-2.83_{-0.1}^{+0.1}$ in this field using a $\sim 13 \mu \mathrm{Jy}^{\text {beam }}{ }^{-1}$ flux density limit. The amplitude for this deep survey suggests less clustering of the fainter sources as expected. However, it needs to be noted that at $3 \mathrm{GHz}$, COSMOS is also likely to look at different population of sources and a direct comparison of the clustering observed in it with the results presented here may not be straight forward.

Hale et al. (2019) reports a clustering amplitude of $\log (\mathrm{A})=$ $-2.08_{-0.05}^{+0.04}$ in the XMM-LSS field at $144 \mathrm{MHz}$. Rana \& Bagla (2019) investigate the clustering for sources in the TIFR GMRT Sky Survey at $150 \mathrm{MHz}$ (TGSS-ADR1) (Intema et al. 2017) over a large fraction of sky and show variation of clustering amplitude as a function of different flux density cut offs. Clustering properties of sources in the Faint Images of the Radio Sky at Twenty-cm (FIRST) survey at $1400 \mathrm{MHz}$ (Becker, White, \& Helfand 1995) is given in Lindsay et al. (2014). The amplitude of the clustering in these surveys are statistically consistent with what we report in this work.

\section{ESTIMATION OF SPATIAL CORRELATION FUNCTION FROM THE REDSHIFT DISTRIBUTION OF SOURCES}

Spatial clustering of sources is quantified by the two-point correlation function $\xi(r)$. With known angular clustering $w(\theta)$ of the sources and the redshift distribution, $N(z)$, it is possible to use the Limber inversion (Limber 1953) method to estimate $\xi(r)$. We briefly discuss this technique here.

Due to gravitational clustering, the spatial clustering of sources 

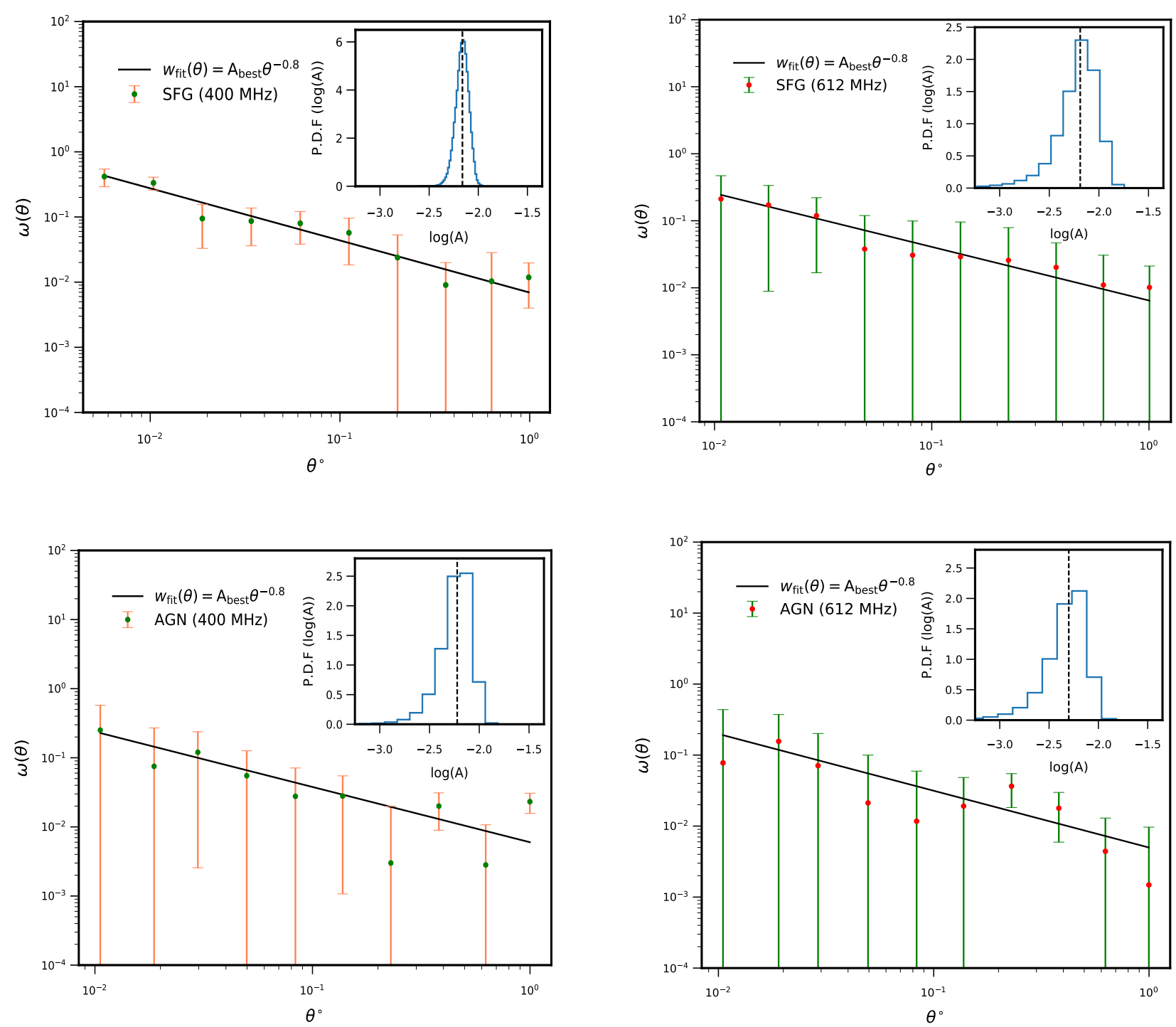

Figure 8. The angular correlation of SFGs (upper panel) and AGNs (lower panel) along with the probability distribution function of amplitude of clustering (insets) when the power-law index $\gamma$ is fixed. The left column is for $400 \mathrm{MHz}$ catalogue and right column is for $612 \mathrm{MHz}$ catalogue.

Table 3. Spatial clustering and bias from this study and previous obsevations

\begin{tabular}{ccccccc}
\hline \hline Observation & Source type & Median redshift & $\log _{10}(\mathrm{~A})$ & $r_{0}$ & $\mathrm{~b}\left(z_{\text {median }}\right)$ & Ref \\
\hline \multirow{2}{*}{ COSMOS } & AGN & 0.70 & $-2.30_{-0.1}^{+0.1}$ & $6.9_{-0.70}^{+0.60}$ & $2.1_{-0.2}^{+0.2}$ & Hale et al. (2018) \\
$(3 \mathrm{GHz})$ & AGN & 1.24 & $-2.60_{-0.1}^{+0.1}$ & $9.6_{-0.70}^{+0.70}$ & $3.6_{-0.2}^{+0.2}$ & Hale et al. (2018) \\
& AGN & 1.77 & $-2.60_{-0.1}^{+0.1}$ & $7.3_{-0.90}^{+0.90}$ & $3.5_{-0.4}^{+0.4}$ & Hale et al. (2018) \\
& SFG & 0.62 & $-2.60_{-0.1}^{+0.1}$ & $5.0_{-0.60}^{+0.50}$ & $1.5_{-0.2}^{+0.1}$ & Hale et al. (2018) \\
& SFG & 1.07 & $-2.90_{-0.1}^{+0.1}$ & $6.1_{-0.70}^{+0.60}$ & $2.3_{-0.2}^{+0.2}$ & Hale et al. (2018) \\
\hline VLA-COSMOS & AGN & 1.25 & $-2.79_{-0.1}^{+0.1}$ & $7.84_{-2.31}^{+1.75}$ & - & Magliocchetti et al. (2017) \\
$(1.4 \mathrm{GHz})$ & SFG & 0.50 & $-2.36_{-0.3}^{+0.3}$ & $5.46_{-2.10}^{+1.12}$ & - & Magliocchetti et al. (2017) \\
\hline ELAIS N1 & AGN & 0.91 & $-2.22_{-0.16}^{+0.16}$ & $7.30_{-1.2}^{+1.4}$ & $3.17_{-0.4}^{+0.5}$ & This work \\
$(400 \mathrm{MHz})$ & SFG & 0.64 & $-2.16_{-0.06}^{+0.05}$ & $4.62_{-0.40}^{+0.39}$ & $1.65_{-0.14}^{+0.14}$ & This work \\
\hline ELAIS N1 & AGN & 0.85 & $-2.30_{-0.03}^{+0.02}$ & $6.0_{-1.3}^{+1.5}$ & $2.6_{-0.5}^{+0.6}$ & This work \\
$(612 \mathrm{MHz})$ & SFG & 0.57 & $-2.18_{-0.02}^{+0.01}$ & $4.16_{-0.8}^{+0.7}$ & $1.59_{-0.2}^{+0.2}$ & This work \\
\hline
\end{tabular}


changes with redshift. We assume a epoch-dependent power-law (Overzier et al. 2003) spatial correlation function as:

$\xi(r, z)=\left(r_{0} / r\right)^{\gamma}(1+z)^{\gamma-(3+\epsilon)}$,

where $\mathrm{r}$ is in comoving units, and $\epsilon$ quantifies different clustering models (Overzier et al. 2003). The parameter $r_{0}$ gives the spatial clustering length at $z=0$. In this work we use comoving clustering model with $\epsilon=\gamma-3$. In this model, the correlation function remains unchanged in comoving coordinates and the cluster has fixed comoving size. The spatial correlation length in comoving coordinates can be estimated as (Peebles 1980):

$A=r_{0}^{\gamma} H_{\gamma}\left(H_{0} / c\right) \frac{\int_{0}^{\infty} N^{2}(z)(1+z)^{\gamma-(3+\epsilon)} \chi^{1-\gamma}(z) E(z) d z}{\left[\int_{0}^{\infty} N(z) d z\right]^{2}}$,

where, $H_{\gamma}=\frac{\Gamma\left(\frac{1}{2}\right) \Gamma\left(\frac{\gamma-1}{2}\right)}{\Gamma\left(\frac{\gamma}{2}\right)}, E(z)=\left[\Omega_{m, 0}(1+z)^{3}+\Omega_{k, 0}(1+z)^{2}+\right.$ $\left.\Omega_{\Lambda, 0}\right]^{1 / 2}, N(z)$ is the redshift distribution of sources and $\chi(z)$ is the line-of-sight comoving distance. We use Eqn. 6 to estimate the spatial correlation length, $r_{0}$, from the estimated clustering amplitude of angular correlation function A, slope of correlation function $\gamma$ and redshift distribution $N(z)$ (Lindsay et al. 2014).

The estimated value of the parameter $\gamma$ across different surveys as mentioned in Table 2 agrees with the theoretical predicted value of 1.8 (Peebles 1980). We use $\gamma=1.8$, the MCMC distribution of the parameter $A$ as given in section 4.1 and the redshift distribution of sources as given in section 3.2 to estimate $r_{0}$. Fig. 7 shows the probability density function for $r_{0}$ in 400 and $612 \mathrm{MHz}$. The median value of $r_{0}$ along with 16 th and 84 th percentile errors are $6.58_{-0.83}^{+0.75}$ at a median redshift of $z=0.803$ for $400 \mathrm{MHz}$ observation. For 612 $\mathrm{MHz}$ observation, the clustering length is $4.19_{-0.74}^{+0.73}$ at a median redshift of $z=0.70$. The lesser value of the clustering length at the $612 \mathrm{MHz}$ is a direct consequence of the lesser clustering of the fainter sources detected at this band.

\subsection{Bias}

The relation between clustering properties of luminous sources and underlying dark matter is described by the bias parameter. The scale independent linear bias parameter $b(z)$ is defined as the ratio of the galaxy to the dark matter spatial correlation functions (Kaiser 1984; Bardeen et al. 1986; Peacock \& Smith 2000). For a standard cosmological model the bias parameter can be calculated from the spatial clustering length $r_{0}$ using (Lindsay et al. 2014; Hale et al. 2018):

$b(z)=\left(\frac{r_{0}(z)}{8}\right)^{\gamma / 2} \frac{J_{2}^{1 / 2}}{\sigma_{8} D(z) / D(0)}$,

were, $J_{2}=72 /\left[(3-\gamma)(4-\gamma)(6-\gamma) 2^{\gamma}\right], D(z)$ is the linear growth factor (Hamilton 2001) and $\sigma_{8}^{2}$ is the dark matter density variance in a comoving sphere of radius $8 \mathrm{Mpc} h^{-1}$. In this work, we have estimated the bias at the median redshift using the median value of $r_{0}$ corresponding to that redshift along with 16 th and 84 th percentile errors. We find that at $400 \mathrm{MHz} b_{z}=2.74_{-0.30}^{+0.27}$ at median redshift of $z=0.803$ and at $612 \mathrm{MHz} b_{z}=1.73_{-0.28}^{+0.27}$ at a median redshift of 0.7 .

\section{CLUSTERING OF SFGS AND AGNS}

In this section, we discuss results of our estimation of the clustering properties and corresponding bias of two different samples contributing to the total source counts, the AGNs and SFGs, at $400 \mathrm{MHz}$ and $612 \mathrm{MHz}$. The evolution of clustering for AGNs and SFGs are expected to be different. Given that the median redshift of the two samples are different in the two observing bands, we expect to trace the hint of redshift evolution of different sources here. Following the similar procedure as described earlier, we estimate angular correlation of AGNs and SFGs and find the best fit value for the amplitude of correlation. The low density of these two samples makes it statistically inappropriate to perform a two parameter fit to the measured correlation function. In view of this, we fix the $\gamma$ to theoretically predicted value of 1.8 and fit for the amplitude $A$ only. Note that the reduced chi square value giving the goodness of the fit for both the SFG ad AGNs are $\sim 0.1$ suggesting that these results has to be taken with caution. However, this analysis allow us to check the consistency of correlation function of SFG's and AGN's with previous findings at radio as well as at other wavelengths. In Fig. 8 , the estimated $w(\theta)$ and the best fit model for SFGs and AGNs are shown. These results are summarized in Table 3. The spatial clustering length for SFGs at a median redshift $z_{\text {median }}=0.64$ is found to be $r_{0}=4.62_{-0.40}^{+0.39} h^{-1} \mathrm{Mpc}$ at $400 \mathrm{MHz}$, which decreases to $r_{0}=4.16_{-0.8}^{+0.7} h^{-1} \mathrm{Mpc}$ at the median redshift of 0.57 at 612 MHz. The corresponding bias for SFGs are $b_{z}=1.65_{-0.14}^{+0.14}$ and $b_{z}$ $=1.59_{-0.2}^{+0.2}$ at $400 \mathrm{MHz}$ and $612 \mathrm{MHz}$ respectively. For the AGN population the clustering length is relatively higher in general. At $z=0.91$ the clustering length for the AGNs is $r_{0}=7.3_{-1.2}^{+1.4} h^{-1}$ $\mathrm{Mpc}$ at $400 \mathrm{MHz}$ which decreases to $r_{0}=6.0_{-1.3}^{+1.5} h^{-1} \mathrm{Mpc}$ at a redshift of $z=0.85$ at $612 \mathrm{MHz}$. The bias for AGNs are $b_{z}=3.17_{-0.4}^{+0.5}$ and $b_{z}=2.6_{-0.5}^{+0.6}$ for 400 and $612 \mathrm{MHz}$ catalogue respectively. We find that there is a hint of decrease of the bias parameter for both populations at higher frequency as the median redshift of these populations decreases. This indicates that we are most likely tracing the fainter less massive haloes at higher frequency. The variation of bias parameter for SFGs and AGNs along with some previous findings are shown in the Fig 10. We also show in Fig. 10, the assumed redshift evolution of bias parameters of different kind of sources in $S^{3}$ simulation. The fact that the estimated bias for AGN is higher compared to that for the SFGs is consistent with results from $S^{3}$ simulation. The large bias parameter indicates that the AGNs are strong biased tracers of dark matter halos than SFGs. Note that, due to uncertainties associated with measurement of redshift, clustering length and bias parameter and owing to the values of almost similar redshifts for different frequency bands, we have not observed much redshift evolution of these different populations here.

\subsection{Comparison to previous observations and discussion}

Compilation of the spatial clustering of AGNs and SFGs at different frequency and redshifts from the literature and this work is summarized in Table 3. The Fig. 9 shows variation of the spatial clustering length as a function of redshift. The left and the right panels show the spatial clustering length for SFGs and AGNs respectively. Note that we only mention the spatial clustering and bias parameters of radio selected samples from previous observations in the Table 3, whereas in Fig. 9 we show previous findings at radio as well as at other frequency surveys. The continuous curves gives the results from the $S^{3}$ simulation for different sub-classes of sources in the $S^{3}$ simulation. The individual markers with error bars corresponds to estimates of the correlation length as measured in this work and from literature.

The clustering length for SFGs found here are similar to the 

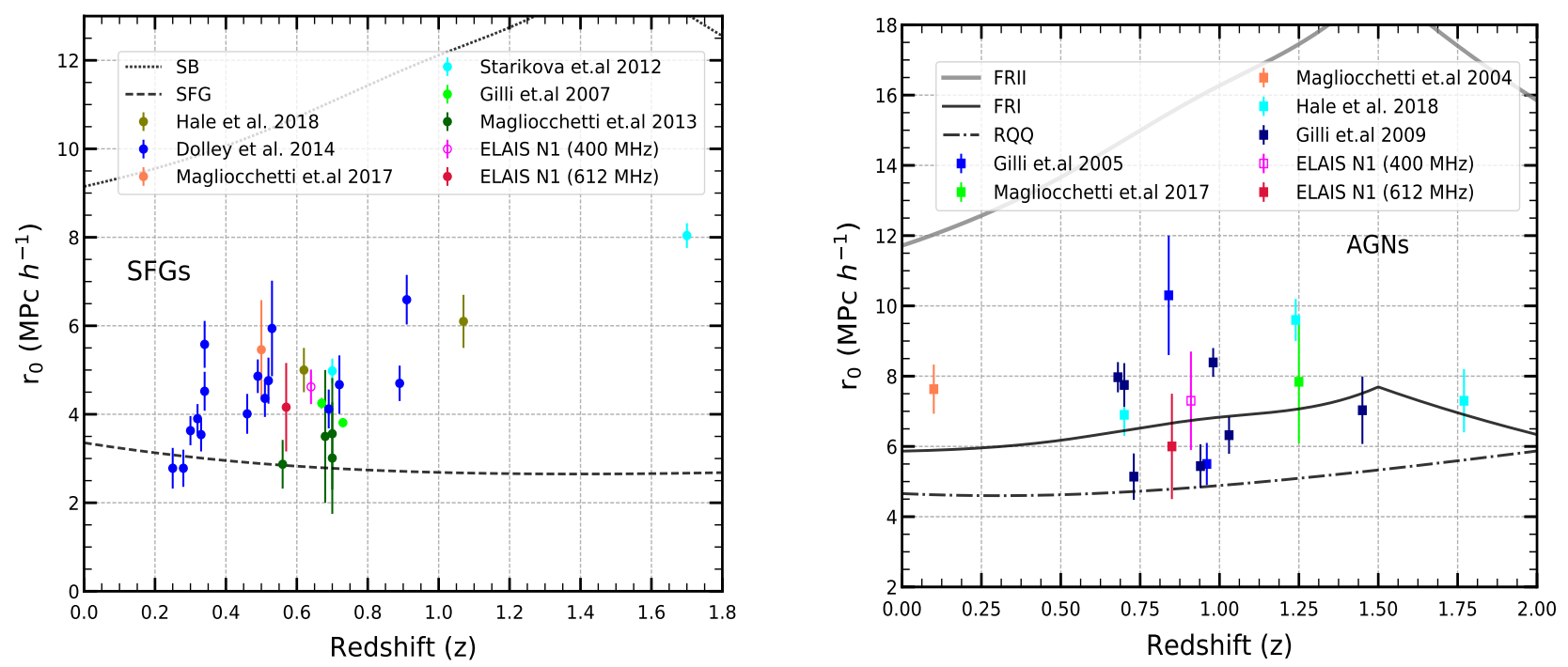

Figure 9. Comparison of the spatial clustering length, $r_{0}(z)$, for SFGs (top right) and AGNs (bottom left) with other surveys taken from literature. The other surveys include: Overzier et al. (2003), Dolley et al. (2014), Magliocchetti et al. (2004), Gilli et al. 2005, Gilli et al. 2007, Gilli et al. 2009, Starikova et al. (2012), Magliocchetti et al. (2013), Lindsay et al. (2014) Magliocchetti et al. (2017), Hale et al. (2018). Solid curves show the evolution of the clustering length as a function of redshift for different source types as obtained from the $S^{3}$ simulation.

previous findings by Hale et al. (2018), Magliocchetti et al. (2017) at redshift $z<1$. Also, the estimated value is close to the clustering length of SFGs selected by different criteria in literature (Gilli et al. 2007; Starikova et al. 2012; Magliocchetti et al. 2013; Dolley et al. 2014; Hale et al. 2018). As mentioned in Hale et al. (2018), the clustering length estimates for SFGs in radio surveys can be little higher than the other wavelength surveys, due to the fact that redshift distribution for SFGs in other wavelength surveys are skewed to lower redshifts. We have seen in Fig. 5 that redshift distribution of SFGs shows a peak at lower redshift and at this redshift the estimated clustering length is in good agreement with other surveys probing the same redshift range (Magliocchetti et al. 2013; Hale et al. 2018). Dolley et al. (2014) have extensively studied the redshift evolution of clustering of SFGs using mid-IR data. They have reported a slow evolution of clustering length as a function of redshift, which is also in agreement with our results.

The spatial clustering length as well as bias parameter of SFGs are higher than $S^{3}$ simulation. This may be arising for two reasons. Firstly, the comparison with the results from $S^{3}$ suggests that, if the SFGs population we study here is contaminated by a few SB galaxies, both the correlation length and the bias parameter is expected to be higher. It may also be the fact that the halo mass in the $S^{3}$ simulation is not a correct representation of halo mass of SFGs and is systematically lower as shown in Fig. 10. The observational evidence of higher halo mass of SFGs in previous studies (Dolley et al. 2014; Magliocchetti et al. 2017; Hale et al. 2018) suggest that the later is the likely cause of the deviation of the two parameters from the $S^{3}$ predictions. In such case, a comparison of bias parameter of SFGs with bias evolution of $S^{3}$ suggests that the halo masses of SFGs at the redshift range probed here are close to $M_{\mathrm{h}} \sim 10^{13} h^{-1} M_{\odot}$ (Fig. 10).

Our result on the clustering of the AGNs statistically similar to (see Figure 9) the earlier reports of the clustering length estimated using radio selected (Magliocchetti et al. 2017) as well as x-ray selected (Gilli et al. 2005, 2009) catalogues. Comparing with the $S^{3}$ simulation suggests that the sources in our catalogue are mostly

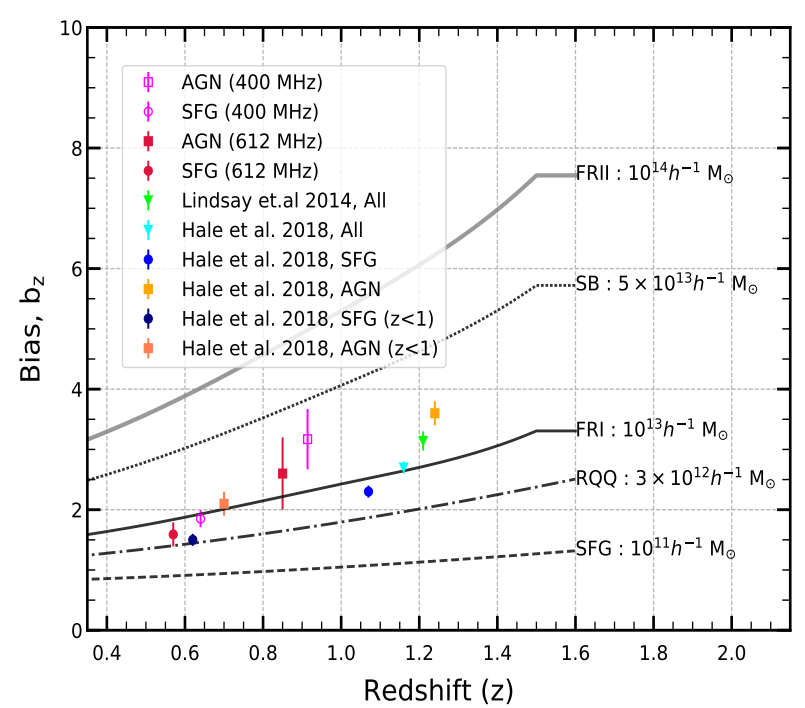

Figure 10. The bias parameter of all sources as well as for SFGs and AGNs are shown for EN1 field as well as in other fields. We also show the bias prescription used for different source populations in SKADS simulation.

of type FRI, which agrees with the earlier results as well. Following the results of the $S^{3}$ we conclude that the AGNs are mostly hosted by massive halos of the order of $M_{\mathrm{h}} \sim 3-4 \times 10^{13} h^{-1} M_{\odot}$ (see Fig. 10). This range of halo masses of AGNs are larger than that of SFGs and emphasizes that fact that AGNs inhabit more massive haloes than SFGs, hence more strong biased tracer of dark matter density field. 


\section{CONCLUSION}

In this work, we investigate the clustering properties of radio sources present in EN1 field at $400 \mathrm{MHz}$ and at $612 \mathrm{MHz}$ and compare it to the the $S^{3}$ simulation as well as previous findings at radio and other wavelengths. We use a luminosity based identifier for radio source populations as AGNs and SFGs. We find the angular correlation functions for all sources in our sample as well as for the SFGs and AGNs individually. The angular correlation function assumes a power law for angular separation ranging $\sim 18^{\prime \prime}-1.5^{\circ}$. The power law index of the correlation function was found to be consistent with 1.8, a value expected from analytical calculations (Peebles 1980). Amplitude of the correlation function indicates that the AGNs are more tightly clustered and are better tracers of the dark matter halos.

We use the BOSS spectroscopic survey and SWIRE photometric survey to add redshift information to the radio sources in our catalogues and estimate the redshift distribution of the different source types. We estimate the spatial clustering length for the sources and the corresponding bias parameters at the representative redshifts.

The clustering length and bias parameter for both samples are in excellent agreement with those found in literature from earlier observations along different lines of sight. Comparing to the results from the $S^{3}$ simulation (Wilman et al. 2008), we found that our AGN population is mostly of type FRI and are hosted by dark matter haloes of halo mass $\sim 3-4 \times 10^{13} h^{-1} M_{\odot}$. We find that the halo mass used for the SFGs in the $S^{3}$ simulation is rather lower and a halo mass of $M_{\mathrm{h}} \sim 10^{13} h^{-1} M_{\odot}$ is likely to host the SFGs.

It is important to note that the radio sources in our catalogue are classified based on radio luminosity only, however, the clustering properties of AGNs/SFGs selected based on X-ray and mid-IR data in other surveys are consistent with our results. We shall present results based on more robust multi-frequency identification of these sources in future.

We compare estimated parameters from our work with observations from earlier surveys. Given the statistical homogeneity and isotropy at the cosmological scales, parameter estimated from different directions in the sky can be compared. It is important to note that the work presented here is the first detailed investigation of the clustering properties of different types of compact radio sources in a given field in multiple frequency bands. We find the evolution of the bias and clustering length over redshift for both AGNs and SFGs as found in the EN1 field agree with earlier results from other different fields observed in radio as well as other wavelengths.

A major science objective of the radio telescopes like LOFAR (van Haarlem et al. 2013) or SKA (Koopmans et al. 2015) is to detect the redshifted $21-\mathrm{cm}$ signal from the neutral hydrogen over a large range of redshifts. Compact extragalactic radio sources are a major source of foreground for the detection of the cosmological 21-cm signal. A major effort has been to understand and characterise the distribution of the compact sources in the radio sky. Moreover, the $21-\mathrm{cm}$ signal being very faint, the radio foreground structures need to be known to low flux density. In this work we use sub mJy source populations sources to estimate their clustering properties. This is the deepest catalogue used to estimate the foreground characteristics at frequencies where the redshifted $21 \mathrm{~cm}$ signal from the post-EoR epoch is expected. Comparing our results with the prediction from $S^{3}$ simulation suggests that their assumption of constant halo mass bias for populating different source types is rather simplistic and needs to be reviewed. Furthermore, this emphasizes the need for further deep studies of foreground characteristics in the low frequencies.
The later also would shed more light on the evolution of the clustering length and bias over redshifts and constrain structure formation models stringently.

\section{ACKNOWLEDGEMENTS}

We would like to thank the anonymous reviewer for suggestions and comments that have helped to improve this paper. AC would like to acknowledge DST for providing INSPIRE fellowship. AC would like to thank Catherine Hale for providing the SKADS bias prescription and for many helpful suggestions. AC would like to thank Matt Jarvis for providing SKADS catalogue over private communication and also for helpful suggestions. AC would like to thank Akriti Sinha for pointing us towards BOSS catalogue for the first time. NR acknowledges support from the Max Planck Society through the Max Planck India Partner Group grant. AD would like to acknowledge the support of EMR-II under CSIR No. 03(1461)/19.

\section{REFERENCES}

Alam S., Ho S., Vargas-Magaña M., Schneider D. P., 2015, MNRAS, 453, 1754

Ali S. S., Bharadwaj S., Chengalur J. N., 2008, MNRAS, 385, 2166

Allison R., et al., 2015, MNRAS, 451, 849

Bandura K., Addison G. E., Amiri M., et al., 2014, SPIE, 9145, 914522

Bardeen J. M., Bond J. R., Kaiser N., Szalay A. S., 1986, ApJ, 304, 15

Barkana R., 2016, PhR, 645, 1

R. A., Brown M. L., Browne I. W. A., et al., 2012, arXiv, arXiv:1209.1041

Becker R. H., White R. L., Helfand D. J., 1995, ApJ, 450, 559

Bernardi G., de Bruyn A. G., Brentjens M. A., et al., 2009, A\&A, 500, 965

Bharadwaj S., Ali S. S., 2005, MNRAS, 356, 1519

Blake C., Wall J., 2002, MNRAS, 337, 993

Chakraborty A., et al., 2019, MNRAS, 490, 243

Chakraborty A., et al., 2019, MNRAS, 487, 4102

Chapman E., Abdalla F. B., Harker G., et al., 2012, MNRAS, 423, 2518

Chapman E., Abdalla F. B., Bobin J., et al., 2013, MNRAS, 429, 165

Chen Z. P., Wang R. L., Peterson J., Chen X. L., Zhang J. Y., Shi H. L., 2016, SPIE, 9906, 99065W

Cole S., et al., 2005, MNRAS, 362, 505

Condon J. J., Cotton W. D., Greisen E. W., Yin Q. F., Perley R. A., Taylor G. B., Broderick J. J., 1998, AJ, 115, 1693

Cooray A., Furlanetto S. R., 2004, ApJ, 606, L5

Cress, C. M., Helfand, D. J., Becker, R. H., Gregg, M. D., White, R. L. 1996. The Angular Two-Point Correlation Function for the FIRST Radio Survey. The Astrophysical Journal 473, 7.

Dawson K. S., et al., 2013, AJ, 145, 10

Dayal P., Ferrara A., 2018, PhR, 780, 1

DeBoer D. R., Parsons A. R., Aguirre J. E., et al., 2017, PASP, 129, 045001

Di Matteo T., Perna R., Abel T., Rees M. J., 2002, ApJ, 564, 576

Di Matteo T., Ciardi B., Miniati F., 2004, MNRAS, 355, 1053

Dolley T. et al., 2014, ApJ, 797, 125

Donoso E., Yan L., Stern D., Assef R. J., 2014, ApJ, 789, 44

Duncan K. J., Jarvis M. J., Brown M. J. I., Röttgering H. J. A., 2018, MNRAS, 477, 5177

Eisenstein D. J. et al., 2011, AJ, 142, 72

Eisenstein D. J. et al., 2005, ApJ, 633, 560

Fan X., Narayanan V. K., Strauss M. A., White R. L., Becker R. H., Pentericci L., Rix H.-W., 2002, AJ, 123, 1247

Fan X., Carilli C. L., Keating B., 2006, ARA\&A, 44, 415

Field G. B., 1958, PIRE, 46, 240

Franzen T. M. O. et al., 2019, PASA, 36, e004

Furlanetto S. R., Oh S. P., Briggs F. H., 2006, PhR, 433, 181

Ghosh A., Prasad J., Bharadwaj S., Ali S. S., Chengalur J. N., 2012, MNRAS, 426, 3295

Gilli R. et al., 2005, A\&A, 430, 811 
Gilli R. et al., 2007, A\&A, 475, 83

Gilli R. et al., 2009, A\&A, 494, 33

Hale C. L., Jarvis M. J., Delvecchio I., Hatfield P. W., Novak M., Smolčić V., Zamorani G., 2018, MNRAS, 474, 4133

Hale C. L. et al., 2019, A\&A, 622, A4

Hamilton A. J. S. 2001, MNRAS, 322, 419

Hatfield P. W., Jarvis M. J., 2017, MNRAS, 472, 3570

Hogg D. W., 1999, arXiv, astro-ph/9905116

Huynh M. T., Jackson C. A., Norris R. P., Prandoni I., 2005, AJ, 130, 1373

Intema H. T., van der Tol S., Cotton W. D., Cohen A. S., van Bemmel I. M., Röttgering H. J. A., 2009, A\&A, 501, 1185

Intema H. T., Jagannathan P., Mooley K. P., Frail D. A., 2017, A\&A, 598, A78

Jarvis M., Bernstein G., Jain B., 2004, MNRAS, 352, 338

Jarvis M., et al., 2016, Proceedings of MeerKAT Science: On the Pathway to the SKA. 25-27 May, 2016 Stellenbosch, South Africa (MeerKAT2016). Available at: https://pos.sissa.it/cgi-bin/ reader/conf.cgi? confid $=277$, id. 6

Jelić V., Zaroubi S., Labropoulos P. et al., 2008, MNRAS, 389, 1319

Jelić V., Zaroubi S., Labropoulos P., Bernardi G., de Bruyn A. G., Koopmans L. V. E., 2010, MNRAS, 409, 164

Kaiser N., 1984, ApJL, 284, L9

Kerscher M., Szapudi I., Szalay A. S., 2000, ApJL, 535, L13

Koopmans L., Pritchard J., Mellema G. et al., 2015, Proceedings of Advancing Astrophysics with the Square Kilometre Array (AASKA14). 9 -13 June, 2014. Giardini Naxos, Italy.Available at: http: //pos.sissa.it/cgi-bin/reader/conf. cgi? confid=215, id.1. (arXiv:1505.07568)

Landy S. D., Szalay A. S., 1993, ApJ, 412, 64

Limber D. N., 1953, ApJ, 117, 134

Lindsay S. N. et al., 2014, MNRAS, 440, 1527

Ling E. N., Frenk C. S., Barrow J. D., 1986, MNRAS, 223, 21P

Loeb A., Furlanetto S. R., 2013, fgu..book, ISBN: 9780691144917 . Princeton, NJ: Princeton University Press

Madau P., 1997, seim.proc, Proceedings of the 13th IAP Astrophysics Colloquium, Institut d'Astrophysique, Paris, France Publisher: Paris: Editions Frontieres, ISBN: 286332229X, 1997., p.295

Magliocchetti M., Maddox S. J., Lahav O., Wall J. V., 1998, MNRAS, 300, 257

Magliocchetti M. et al., 2002, MNRAS, 333, 100

Magliocchetti M. et al., 2004, MNRAS, 350, 1485

Magliocchetti M. et al., 2013, MNRAS, 433, 127

Magliocchetti M., et al., 2014, MNRAS, 442, 682

Magliocchetti M., Lutz D., Santini P., Salvato M., Popesso P., Berta S., Pozzi F., 2016, MNRAS, 456, 431

Magliocchetti M., Popesso P., Brusa M., Salvato M., Laigle C., McCracken H. J., Ilbert O., 2017, MNRAS, 464, 3271

McAlpine K., Jarvis M. J., Bonfield D. G., 2013, MNRAS, 436, 1084

McMullin J. P., Waters B., Schiebel D., Young W., Golap K., 2007, ASPC..376, 127, ASPC..376

Mesinger A., 2016, ASSL, 423

Mo H. J., White S. D. M., 1996, MNRAS, 282, 347

Mohan N., Rafferty D., 2015, ascl.soft, ascl:1502.007

Newburgh L. B., Bandura K., Bucher M. A., et al., 2016, SPIE, 9906, 99065X

Norris R. P. et al., 2011, PASA, 28, 215

Offringa A. R., van de Gronde J. J., Roerdink J. B. T. M., 2012, A\&A, 539, A95

Oort M. J. A., 1987, PhD thesis, Leiden Observatory

Overzier R. A., Röttgering H. J. A., Rengelink R. B., Wilman R. J., 2003, A\&A, 405, 53

Peacock J. A., Smith R. E., 2000, MNRAS, 318, 1144

Peebles P. J. E., 1980, 1ssu.book

Planck Collaboration, et al., 2014, A\&A, 571, A17

Planck Collaboration et al., 2018, arXiv, arXiv:1807.06209

Planck Collaboration et al., 2016, A\&A, 596, A108

Pober J. C., Parsons A. R., DeBoer D. R., et al., 2013, AJ, 145, 65
Prandoni I., Guglielmino G., Morganti R., Vaccari M., Maini A., Röttgering H. J. A., Jarvis M. J., Garrett M. A., 2018, MNRAS, 481

Press W. H., Schechter P., 1974, ApJ, 187, 425

Pritchard J. R., Loeb A., 2012, RPPh, 75, 086901

Raccanelli A. et al., 2015, JCAP, 2015, 042

Rana S., Bagla J. S., 2019, MNRAS, 485, 5891

Rowan-Robinson M. et al., 2008, MNRAS, 386, 697

Rowan-Robinson M., Gonzalez-Solares E., Vaccari M., Marchetti L., 2013, MNRAS, 428, 1958

Seymour N. et al., 2007, ApJS, 171, 353

Seljak U., 2009, PhRvL, 102, 021302

Shaver P. A., Windhorst R. A., Madau P., de Bruyn A. G., 1999, A\&A, 345, 380

Shimwell T. W. et al., 2017, A\&A, 598, A104

Smolčić V. et al., 2017, A\&A, 602, A2

Smolčić V. et al., 2017, A\&A, 602, A1

Starikova S., Berta S., Franceschini A., Marchetti L., Rodighiero G., Vaccari M., Vikhlinin A., 2012, ApJ, 751, 126

Taylor A. R., Jagannathan P., 2016, MNRAS, 459, L36

Trott C. M., Pindor B., Procopio P. et al., 2016, ApJ, 818, 139

van Haarlem M. P., Wise M. W., Gunst A. W., et al., 2013, A\&A, 556, A2

Wilman R. J., Miller L., Jarvis M. J. et al., 2008, MNRAS, 388, 1335

Wyithe J. S. B., Loeb A., 2008, MNRAS, 383, 606

York D. G. et al., 2000, AJ, 120, 1579

Zaroubi S., de Bruyn A. G., Harker G. et al., 2012, MNRAS, 425, 2964

Zaldarriaga M., Furlanetto S. R., Hernquist L., 2004, ApJ, 608, 622

This paper has been typeset from a $\mathrm{T}_{\mathrm{E}} \mathrm{X} / \mathrm{L} \mathrm{AT} \mathrm{E} \mathrm{X}$ file prepared by the author. 